\title{
Modelo explicativo de la rentabilidad económica del cultivo de durazno en la provincia de Pamplona, Colombia ${ }^{1}$
}

\section{Explanatory model of the economic profitability of peach crop in the Province of Pamplona, Colombia}

\author{
DOI: http://dx.doi.org/10.17981/econcuc.39.2.2018.04
}

Artículo de investigación. Fecha de recepción: 23/05/2018 Fecha de aceptación: 02/10/2018

\author{
Susan Cancino \\ Universidad de Pamplona (Colombia). \\ susancancino@unipamplona.edu.co
Universidad de Pamplona (Colombia). gcancino@unipamplona.edu.co \\ Giovanni Cancino Escalante

\section{Enrique Quevedo García iD \\ Universidad de Pamplona (Colombia). \\ enriquegarcia@unipamplona.edu.co}

Para citar este artículo:

Cancino, S., Cancino, G. y Quevedo, E. (2018). Generación de valor: factor clave en la toma de decisiones de las pymes. Económicas CUC, 39(2). 63-76. DOI: http://dx.doi.org/10.17981/econcuc.39.2.2018.04

\section{Resumen}

En Colombia, el departamento de Norte de Santander, en el cual se ubica la provincia de Pamplona, es el segundo mayor productor de durazno del país posicionándose como un cultivador de vocación frutícola. El objetivo de esta investigación es determinar un modelo econométrico explicativo de la rentabilidad económica para veintisiete (27) fincas productoras de durazno con la finalidad de evaluar cómo las variables costo de producción, rendimiento, ingresos y edad del cultivo afectan la rentabilidad económica de estos cultivadores. La investigación se basó en un estudio de campo, metodología de corte transversal; se utilizó una encuesta estructurada y se efectuaron pruebas de validez. Los resultados mostraron que las variables propuestas explican la variación en la rentabilidad económica, y que el modelo no presenta problemas de normalidad, colinealidad, heteroscedasticidad y autocorrelación.

Palabras clave: Modelo de regresión, rentabilidad económica, prueba de validez

\section{Abstract}

The department of North of Santander (Colombia), where the Province of Pamplona is located, is the country's second peach producer. The objective was to determine an explanatory economic profitability model for twenty seven (27) peach producers, in order to evaluate how the variables production cost, yield, income and crop age affects economic profitability. The research was based on field studies and a cross-sectional methodology was used. A structured survey was conducted, once the data was analyzed, and the validity tests were carried out results showed that the proposed variables explained the variations in the economic profitability and that the model did not present problems of normality, collinearity, heteroscedasticity and autocorrelation, therefore in accordance with the proposed model.

Keywords: Regression model, economic profitability, validity test

1 Artículo científico derivado de la investigación "Aporte al estudio de la huella genómica mediante marcadores moleculares SSR de Prunus persica (Var. amarillo Jarillo y gran jarillo), y el patógeno Monilinia fruticula en cultivos comerciales de durazno en la Provincia de Pamplona”. 


\section{Introducción}

El durazno [P. persica (L.) Batsch] es una de las especies de frutales caducifolias que más se cultivan tanto en las zonas templadas (latitudes entre $30^{\circ}$ a $55^{\circ}$ ) en el hemisferio norte, como en las zonas de alta montaña de los trópicos y subtrópicos $\left(30^{\circ}\right.$ a $45^{\circ}$ ) en el hemisferio sur. Es originario de China y fue introducido a Persia (actualmente Irán) por los comerciantes de seda, luego llevado a Europa, para finalmente los conquistadores españoles en el siglo XV a Latino América (Pinzón, Cruz y Fischer, 2014). El cultivo del durazno es una de las actividades que más se ha incrementado en relación con otros frutales caducifolios en el mundo. El mayor productor es China, seguido de Italia y Estados Unidos; y en Latinoamérica, Chile con 97.564,5 toneladas por año, seguido de México, Brasil y Colombia (Africano, Almanza-Merchón, Criollo, Herrera y Balaguera-López, 2016).

Patiño y Miranda (2013) indican que en Colombia las zonas de producción de durazno están en diferentes ecorregiones del país, presentando diversidad de cultivares en su mayoría de origen desconocido. Adicionalmente los niveles de tecnología empleados no son uniformes entre las regiones, entre las mismas fincas y los sistemas de producción, igualmente presentan variaciones y niveles de desarrollo contrastantes. Sin embargo, por su ubicación geográfica y sus diferentes microclimas combinados con el buen manejo agronómico se pueden producir duraznos durante todo el año en Colombia (Cárdenas y Fischer, 2013).

La producción agronómica de durazno en Colombia se concentra en los departamentos andinos de Boyacá, Cundinamarca, Norte de Santander y Santander sobre la cordillera oriental; de los cuales los dos primeros producen las variedades Dorado, Diamante Rubidoux y Rey Negro mientras que en el Norte de Santander y Santander las variedades producidas son Jarillo y Gran Jarillo (Campos, 2013).
Según datos del Ministerio de Agricultura (República de Colombia. Minagricultura, 2014) en el año 2014 la producción total nacional de durazno fue de 26.114 toneladas (ton) del cual el departamento del Norte de Santander participó con el $36,85 \%$ (9.622 ton), siendo el segundo mayor productor. Con relación al área sembrada, el departamento viene presentando un incremento pasando de 168 a 830 hectáreas (ha) en el periodo 2007-2014 distribuidas de la siguiente forma: en el municipio de Pamplonita 82,3 ha, en Pamplona 43,6 ha, en Chitagá 236,5 ha, en Cácota 210,5 ha y en el municipio de Silos 170,8 ha (Quevedo, Darghan y Fischer, 2017), posicionándose, así, cómo un departamento con una gran vocación frutícola.

En la provincia de Pamplona, situada en el departamento del Norte de Santander, el durazno es un producto de gran importancia en la economía agrícola, especialmente para los municipios de Cácota y Silos (Quevedo et al., 2017). La explotación del cultivo es en su mayoría llevada a cabo por pequeños productores que poseen parcelas de uno a dos hectáreas; el $76,3 \%$ son propietarios de la tierra y los $23,77 \%$ restantes se dividen entre aparcería y contratos; la cosecha es en promedio de 30 días y el $95 \%$ se realiza de forma manual (Puentes, Bastidas y Lemus, 2015; Villamizar, 2008). Asimismo, la comercialización del durazno se hace principalmente con fruta fresca como materia prima para el mercado nacional de jugos naturales, enlatados y conservas (Peñaranda, 2012).

Por consiguiente, el cultivo del durazno es un factor dinamizador de la economía de la región productora, tanto para los actores primarios como para los servicios relacionados; es una importante fuente de ingresos para el sostenimiento de los agricultores; intensiva en factor trabajo, y además, utiliza mano de obra local lo cual contribuye a la reducción de la migración de la población rural hacia las grandes concentraciones urbanas. 
En este contexto, es de vital importancia efectuar un estudio de la rentabilidad económica del cultivo del durazno y, más aún, cuando son prácticamente pocos los trabajos publicados al respecto. En consecuencia, es clave determinar la relación existente entre los ingresos obtenidos y los gastos incurridos en el proceso de producción, ya que mediante este análisis se pueden determinar los beneficios económicos resultado de la inversión; debido a que se considera una inversión a largo plazo, dado que a partir de su siembra o trasplante tarda entre 2 y 3 años para alcanzar una producción estable (Fischer, 2013).

Para llevar a cabo este análisis se efectua el planteamiento de un modelo matemático para evaluar la rentabilidad económica del cultivo de durazno de veintisiete (27) fincas localizadas en la provincia de Pamplona (Colombia), permitiendo determinar cuáles fueron los factores y elementos que poseen mayor influencia en la misma. Este enfoque es importante debido a la inexistencia en la actualidad de análisis sobre este tema en la provincia de Pamplona, lo cual permite obtener resultados confiables; para, así, optimizar el uso de los recursos en forma eficiente y establecer las maneras de actuar del productor para no incurrir en perdidas, y servirá para que nuevos productores decidan invertir en este cultivo, así mismo, en términos económicos logren un mayor excedente al producir durazno.

\section{Antecedentes}

Existen diversos conceptos relacionados con el término rentabilidad, siendo este uno de los indicadores más utilizados para evaluar el éxito o fracaso de un sector o negocio. Faga y Ramos (2006) la define como una ganancia o utilidad, es decir, el excedente del ingreso sobre los costos totales relacionados con la actividad productiva del negocio; mientras Aguirre, Prieto y Escamilla (1997) la consideran como un beneficio importante que debe ser alcanzado en el corto plazo para el desarrollo óptimo de un negocio.

En términos más concisos, la rentabilidad es una herramienta importante para dirigir las actividades diarias de los productores como parte de sus funciones, una vez que proporciona un punto de referencia con el cual todas las operaciones pueden ser medidas. Así, la importancia del análisis de rentabilidad según Ramírez (2008), se centra entre la rentabilidad y seguridad como variables fundamentales de una actividad económica.

Por otra parte, Rivero y Cuervo (1986) afirman que el estudio de la rentabilidad se puede realizar en función del tipo de resultado, por lo cual define como rentabilidad económica, que tiene como objetivo medir la eficacia del negocio; y en función de la inversión, determinado por la rentabilidad financiera, cuya función es medir la capacidad de la empresa para crear riqueza a favor de los propietarios.

En este contexto el estudio se basa en la rentabilidad económica de la actividad productiva porque de ésta depende el logro de sus objetivos a corto, mediano y largo plazo, dado que, la maximización de los beneficios se obtiene sobre la base de los términos económicos (Ramírez, 2008). Por consiguiente, para determinar la rentabilidad económica es necesario comprender los costos en que incurren los productores, de los cuales pueden variar de acuerdo al establecimiento y al manejo de las diferentes unidades productivas.

Faga y Ramos (2006) conceptualiza el costo como "el insumo de determinados elementos valorizables económicamente, aplicado a lograr un objetivo también económico". Según Varían (2016), se entiende por costo "los gastos que incurren las unidades productivas cuando compran factores para producir bienes que planean vender". $\mathrm{Pa}$ ra Ramírez y Vanegas (2008) los costos a considerar en el proceso productivo son importantes para la determinación del punto 
de equilibrio, es decir el punto mínimo de ganancias, una vez que permite decisiones oportunas y adecuadas de inversión. En este sentido, ante las variaciones en los costos de los factores de producción, la rentabilidad económica se verá afectada, es decir, cuanto mayor los costos de producción menor será el margen de ganancias de las unidades productivas.

Por otra parte, existe igualmente un fuerte grado de complementariedad entre los ingresos de las unidades productivas y el indicador de la rentabilidad económica. Según Faga y Ramos (2006), el ingreso se refiere a la cantidad que recibe una empresa por la venta de su producción, por lo cual asegura las actividades de la misma en el presente y a la vez promueve su desarrollo futuro. Igualmente, para Ramírez (2008) el desempeño de una empresa se puede evaluar en función de su ingreso, si está ganando o perdiendo su rentabilidad económica se verá afectada de forma positiva o negativa, es decir, el margen de ganancia aumentará o disminuirá, respectivamente.

En vista de lo anterior, desde la perspectiva económica, la rentabilidad de una unidad productiva está influida por la eficiencia de la misma; así, una mayor rentabilidad pasa por una gestión eficiente de los costos de producción, es decir la minimización de los costos y un aumento del ingreso (Varían, 2016). En consecuencia, para planear y gestionar de manera efectiva las unidades productivas es necesario realizar los respectivos análisis desde el punto de vista de la rentabilidad económica.

\section{Metodología}

La investigación se desarrolla en la provincia de Pamplona, (Norte de SantanderColombia), en 27 fincas productoras, en los municipios de Pamplona, Cacota y Silos, cuya altitud se encuentra entre los $1.850 \mathrm{y}$ 2.170 msnm (Tabla 1).

Para la recolección de los datos se aplica una encuesta estructurada a veintisiete (27) productores de durazno ( $P$. persica), con la finalidad de obtener información sobre los costos de producción por hectárea $\left(\$ / \mathrm{ha}^{-1}\right)$, el rendimiento por hectárea $\left(\mathrm{kg} / \mathrm{ha}^{-1}\right)$, el ingreso obtenido por la venta del producto por hectárea $\left(\$ / \mathrm{ha}^{-1}\right)$ y la rentabilidad económica en términos porcentuales (\%) para cada unidad productiva, de acuerdo al modelo propuesto por Ramírez y Ávila (2013). Sin embargo, en el presente estudio también se consideró la edad del cultivo en años por ser un factor importante que incide en la producción (Cárdenas y Fischer, 2013).

La variable costos de producción esta compuesta por los elementos de gastos de insumos, equipos y mano de obra para el establecimiento y sostenimiento del cultivo. Con relación al cálculo del rendimiento del cultivo se considera el número de árboles de durazno, el área sembrada, la distancia entre los árboles y la producción por cada planta; datos proporcionado por los productores, mientras que para la obtención de los ingresos se utilizaron los precios promedio de venta de las diferentes calidades del cultivo de durazno, las cantidades de árboles por hectárea y sus respectivas producciones.

TABLA 1

Ubicación de las localidades de muestreo Pamplona, Silos y Cacota.

\begin{tabular}{|c|c|c|c|}
\hline \multirow{2}{*}{$\begin{array}{l}\text { Municipio y corregimiento de } \\
\text { la Provincia de Pamplona }\end{array}$} & \multicolumn{2}{|c|}{ Coordenadas } & \multirow{2}{*}{$\begin{array}{c}\text { Altura sobre el } \\
\text { nivel del mar } \\
\text { msnm }\end{array}$} \\
\hline & Latitud & Longitud & \\
\hline Pamplona & $7^{\circ} 22^{\prime} 43,6 ” \mathrm{~N}$ & $72^{\circ} 37^{\prime} 411^{\prime \prime} \mathrm{W}$ & 2.170 \\
\hline Silos & $7^{\circ} 18^{\prime} 33,3^{\prime \prime} \mathrm{N}$ & $72^{\circ} 73^{\prime} 33^{\prime \prime} \mathrm{W}$ & 1.850 \\
\hline Cacota & $7^{\circ} 16^{\prime} 04^{\prime \prime} \mathrm{N}$ & $72^{\circ} 38^{\prime} 30^{\prime \prime} \mathrm{W}$ & 1.890 \\
\hline
\end{tabular}

Fuente: elaboración propia. 
En el cálculo de la rentabilidad económica de cada unidad productiva se utiliza la relación ingresos/costos como indicador de viabilidad económica, basados en la teoría microeconómica (Varían, 2016), del cual fue evaluada mediante la siguiente ecuación matemática:

Rendimiento $=$ (Ingreso-Costo de Producción $) \times 100$ Económico Costo de Producción

Se hicieron uso de herramientas econométricas a través del paquete E-Views ${ }^{\circledR} 9$ (2016) para el análisis, interpretación y presentación del modelo propuesto. De igual modo, los parámetros fueron estimados mediante una regresión lineal múltiple utilizando el método de Mínimos Cuadrados Ordinarios (MCO).

\section{Especificación del Modelo}

Dado que el objetivo del presente estudio se centra en explicar la relación entre las variables exógenas; Costos de Producción (CP), Rendimiento (REND), Ingreso (I) y Edad del Cultivo (EDAD) sobre la variable endógena; Rentabilidad económica (RENT), se plantea el siguiente modelo de regresión lineal basado en Ramírez y Ávila (2013):

$\mathrm{RENT}=81-82 \mathrm{CP}++$ B3REND + B4I + B5EDAD $+\mu$

Los signos que aparecen en la ecuación indican el sentido esperado del efecto de las variables independientes sobre la rentabilidad económica del cultivo de durazno. En este sentido, el signo del coeficiente 82 es negativo, esto es, corresponde a una relación inversa entre los costos de producción y la rentabilidad económica dado que un aumento en los costos reduce los beneficios económicos de los productores.

Por otra parte, para la variable ingreso se considera que la misma posee una relación directa, debido a que un incremento en el ingreso afecta positivamente la rentabilidad del cultivo, es decir, esta será más alta cuanto mayor sea el ingreso obtenido por la venta del producto y menor sea su costo de producción.
Con respecto a la variable edad, existe igualmente una relación positiva con la rentabilidad económica, una vez que el durazno es un árbol que empieza a producir entre el segundo y tercer año, y en el caso del presente estudio se identifica que las veintisiete (27) fincas poseen árboles de durazno con una edad promedio de 4 años, lo cual significa que se encuentran en una etapa ascendente de producción.

\section{Resultados}

A pesar de la importancia que tiene para el desarrollo del sector agrícola, los modelos explicativos sobre la rentabilidad económica de los cultivos son escasos. La mayoría de los estudios se enfocan en la relación entre los costos de producción y el ingreso con la rentabilidad a través del cálculo del indicador y las variables que componen el mismo (Peñaranda, 2012).

Sin embargo, Flórez y Miranda (2017), cuya investigación se basa en determinar un modelo para medir la rentabilidad de los cereales alto andino. Ramírez y Ávila (2013) en sus estudios sobre los factores que influyen en la rentabilidad económica de la producción del cultivo de camu camu en la selva peruana, al igual que la presente investigación, utilizaron herramientas econométricas para determinar y analizar cuales variables influyen sobre la rentabilidad económica del cultivo de durazno.

\section{Estimación de los parámetros}

Tomando como referencia los datos obtenidos de la encuesta realizada a los 27 productores de durazno de la provincia de Pamplona, los resultados de la regresión lineal por el método MCO se muestran en la Tabla 2. Se puede observar que el valor de los coeficientes de regresión parcial y sus respectivos niveles de significatividad individuales son superiores a $95 \%$, siendo el de mayor influencia el costo de producción (t-estadístico $=|11,67865|$ ). Por otra 
parte, el intercepto, el cual no posee relevancia económica (Gujarari y Porter, 2010), no es estadísticamente significativo, por lo cual se rechaza su influencia sobre el comportamiento de la rentabilidad económica.

Como se puede inferir, el estadístico $\mathrm{F}=$ 59,013 , con una probabilidad de ocurrencia igual a $0,000(\mathrm{p}<0,05)$, señala que conjuntamente las variables regresoras son estadísticamente significativas. El coeficiente de determinación $\left(\mathrm{R}^{2}\right)$ indica que $91 \%$ de las variaciones en la rentabilidad son explicadas por las variables regresoras consideradas en el modelo, para un total de 27 observaciones.

\section{Pruebas de diagnóstico}

Una vez estimado los parámetros del modelo de regresión lineal, se procede a realizar un conjunto de pruebas para evaluar la validez de los resultados obtenidos. Inicialmente se utilizó el histograma de los residuos (un dispositivo gráfico) y la prueba de JarqueBera (JB) (Jarque y Bera, 1987) con el fin de verificar si el término del error sigue una distribución normal, una vez que esta comprobación permite que los procedimientos estadísticos utilizados en el estudio sean los correctos (Gujarati y Porter, 2010). Además, la utilización de dicha prueba ha demostrado una consistencia cuanto al correcto análi- sis de los resultados (Thadewald y Buning, 2007).

A partir del histograma se observa (Figura 1) que el residuo está normalmente distribuido y que el valor del estadístico (JB) es de 3,32, menor que el valor crítico tabulado $\left(\mathrm{X}^{2}=5,99\right)$ para un nivel de significación del 5\%; al mismo tiempo la probabilidad asociada es de 0,18 ( $p>0,05)$, lo cual concluye que el supuesto de normalidad de los errores para el modelo propuesto se cumple.

Posteriormente se analiza la posible existencia de multicolinealidad (una relación lineal entre las variables explicativas); un problema frecuente en los modelos de regresión que tiene como consecuencia la imposibilidad de medir la incidencia de cada variable exógena sobre la variable endógena (Gujarti y Porter, 2010). Para su detección se utilizaron algunas reglas prácticas como la evaluación del coeficiente de determinación $\left(\mathrm{R}^{2}\right)$ y el método aplicado por Kleinbaum, Kupper y Muller (2014), en el cual se asume que existe un problema de colinealidad cuando la regresión auxiliar de las variables explicativas (la regresión de cada variable independiente sobre las restantes variables independientes) poseen un $\mathrm{R}^{2}$ mayor que la regresión global y algún Factor Inflacionario de la Varianza (FIV) es superior a 10.

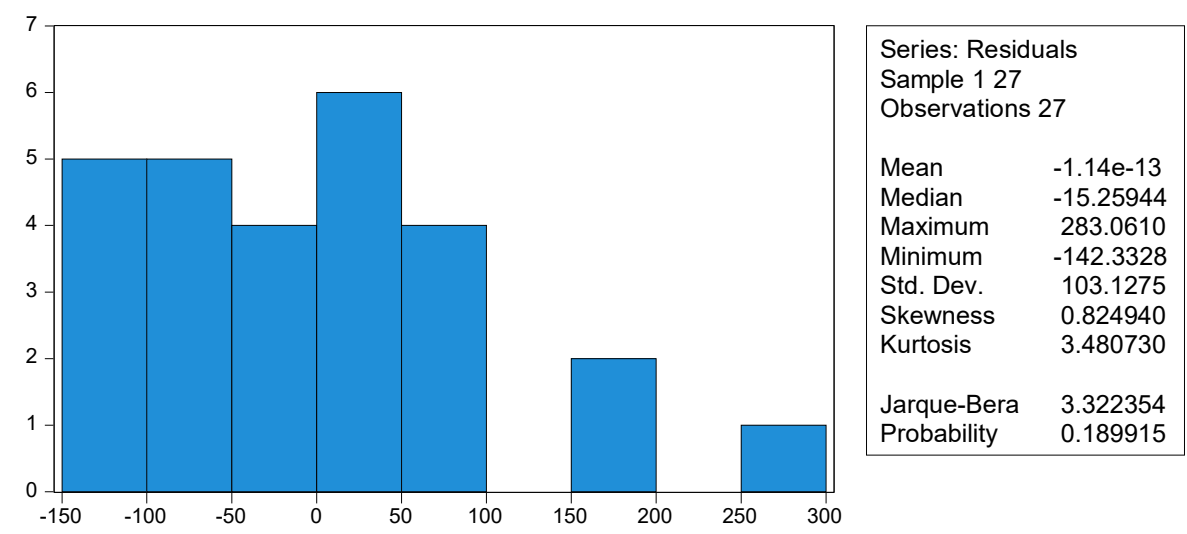

Figura 1. Histograma de los residuos y Prueba Jarque-Bera

Fuente: elaboración propia a partir de E-views®9 (2016). 
TABla 2

Resultados de la estimación del modelo por MCO

\begin{tabular}{|c|c|c|c|c|}
\hline \multicolumn{5}{|c|}{ Variable dependiente: RENT } \\
\hline \multicolumn{5}{|c|}{ Método mínimos cuadrados } \\
\hline Observaciones 1 & & & & \\
\hline Variable & Coeficiente & Error Estándar & t-estadístico & Probabilidad \\
\hline $\mathrm{CP}$ & $-4,84 \mathrm{E}-05$ & $4,15 \mathrm{E}-06$ & $-11,67865$ & 0,0000 \\
\hline REND & 0,008916 & 0,004003 & 2,227278 & 0,0365 \\
\hline I & $6,68 \mathrm{E}-06$ & $2,00 \mathrm{E}-06$ & 3,343346 & 0,0029 \\
\hline EDAD & 71,59720 & 27,95485 & 2,561173 & 0,0178 \\
\hline $\mathrm{C}$ & 74,18790 & 110,5592 & 0,671024 & 0,5092 \\
\hline $\mathrm{R}^{2}$ & 0,914746 & & & \\
\hline F-statistic & 59,01340 & & & \\
\hline Prob(F-statistic) & 0,000000 & & & \\
\hline
\end{tabular}

Fuente: elaboración propia a partir de E-views®9 (2016).

TABLA 3

Coeficientes de determinación del modelo global y parcial

\begin{tabular}{|c|c|c|}
\hline Modelo & Especificación & $\mathbf{R}^{2}$ \\
\hline Global & $\mathrm{RENT}=B_{1}-B_{2} \mathrm{CP}+B_{3} \mathrm{REND}+B_{4} \mathrm{I}+B_{5} \mathrm{EDAD}+\mu$ & 0,91 \\
\hline Regresión 1 & $\mathrm{CP}=B_{1}+B_{2} \mathrm{REND}+B_{3} \mathrm{I}+\beta_{4} \mathrm{EDAD}+\mu$ & 0,17 \\
\hline Regresión 2 & $\mathrm{REND}=B_{1}-B_{2} \mathrm{CP}+B_{3} \mathrm{I}+B_{4} \mathrm{EDAD}+\mu$ & 0,84 \\
\hline Regresión 3 & $\mathrm{I}=B_{1}-B_{2} \mathrm{CP}+\beta_{3} \mathrm{REND}+\beta_{4} \mathrm{EDAD}+\mu$ & 0,85 \\
\hline Regresión 4 & $\mathrm{EDAD}=81-B_{2} \mathrm{CP}+B_{3} \mathrm{I}+B_{4} \mathrm{EDAD}+\mu$ & 0,10 \\
\hline
\end{tabular}

Fuente: elaboración propia.

El resultado de la regresión nos muestra un coeficiente de determinación $\mathrm{R}^{2}=0,91$ sugiriendo un problema de colinealidad (Tabla 2). Sin embargo, cuando aplicamos la regla de Kleinbaum, observamos que los modelos parciales no poseen un coeficiente de determinación superior al modelo global (Tabla 3) además, al calcular el FIV (Tabla 4) de cada variable comprobamos que ninguna presenta un valor superior a 10 , por lo que podemos concluir que no existe multicolinealidad en el modelo propuesto.
TABLA 4

Valores FIV obtenidos de las regresiones parciales

\begin{tabular}{cc}
\hline Variable dependiente & FIV \\
\hline CP & 1,20 \\
I & 6,25 \\
REND & 6,66 \\
EDAD & 1,11 \\
\hline \hline
\end{tabular}

Fuente: elaboración propia. 
Igualmente, se supone que en un modelo de regresión lineal no debe existir una autocorrelación en los errores, es decir, "el término del error relacionado con una observación no recibe influencia del término de error relacionado con otra observación" (Gujarati y Porter, 2010, p. 413). Por consiguiente, con la finalidad de evaluar la presencia de autocorrelación se utilizó el correlograma Q-Statistic (Figura 2), donde se observa la no existencia de un valor Prob inferior a 0,05 , lo que indica que podemos rechazar la hipótesis de presencia de autocorrelación en el modelo.
Por otra parte, para comprobar la existencia de heterescedasticidad, es decir, cuando la varianza del error no es constante, sin importar los valores que toman las variables explicativas, se utilizó la prueba de White con y sin términos de los productos cruzados (Tabla 5). A la vista de estos resultados observamos que en ambos casos la probabilidad asociada al F-estadístico y al Chi-cuadrado es superior a 0,05, lo cual aceptamos la hipótesis nula, confirmando, así, que el modelo propuesto no presenta problemas de heteroscedasticidad a un nivel de confianza de $95 \%$.

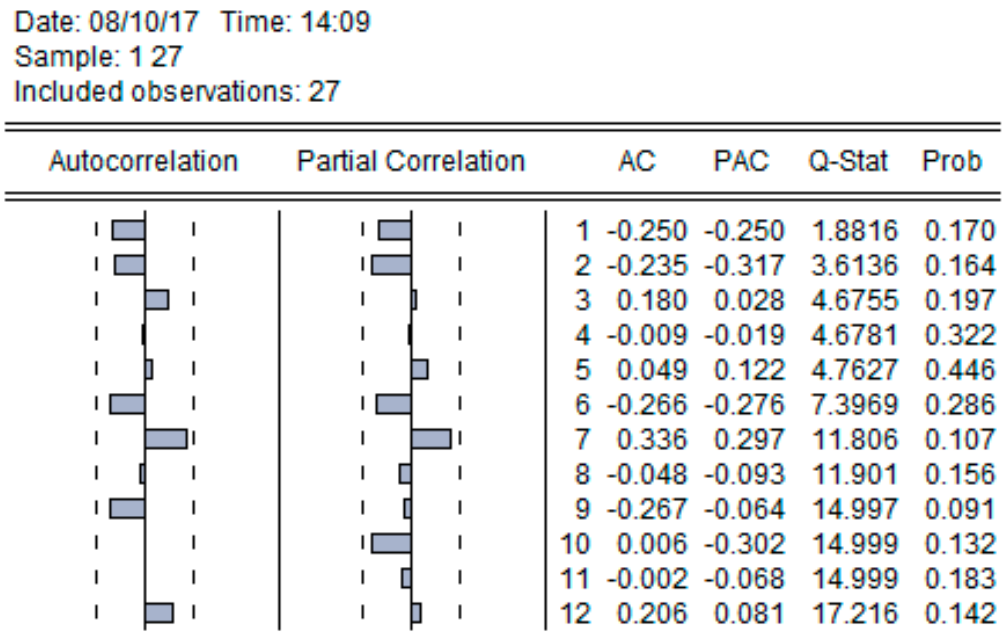

Figura 2. Correlograma de los Residuos.

Fuente: elaboración propia a partir de E-views®9 (2016).

TABLA 5

Test de White Términos cruzados y no cruzados

\begin{tabular}{llll}
\hline \hline Términos Cruzados & & & \\
\hline F-estadístico & 1,402667 & Probabilidad. & 0,2815 \\
Chi-cuadrado & 16,75894 & Probabilidad & 0,2693 \\
$\begin{array}{l}\text { Términos no } \\
\text { cruzados }\end{array}$ & & & \\
$\begin{array}{l}\text { F-estadístico } \\
\text { Chi-cuadrado }\end{array}$ & 1,143117 & Probabilidad & 0,3624 \\
\hline
\end{tabular}

Fuente: elaboración propia a partir de E-views ${ }^{\circledR} 9$ (2016). 
Pruebas de estabilidad de los parámetros

Una de las hipótesis básicas en econometría se refiere a la estabilidad de los parámetros del modelo de regresión, el cual asume que los estimadores permanecen constantes a lo largo de la muestra. El rechazo de dicha hipótesis indica que el valor de los estimadores no mide adecuadamente la relación entre las variables y genera un sesgo en la distribución de los errores, por consiguiente, eliminando la capacidad predictiva del modelo (Gómez y Rodríguez, 2007). Es por ello que, con la finalidad de examinar la estabilidad de los estimadores se recorrió a las pruebes CUSUM y CUSUM al cuadrado (CUSUMQ).
En la Figura 3 se puede apreciar un comportamiento creciente y estable para la totalidad de las observaciones con relación a las dos pruebas efectuadas, asimismo, ninguno de los residuos sobrepasa las bandas de confianza construidas en torno a la significancia del $5 \%$. En consecuencia, se puede concluir que existe estabilidad de los parámetros en el modelo propuesto.

\section{Pruebas de estacionariedad y de Granger}

De igual forma se efectuaron pruebas con la finalidad de detectar la existencia de estacionariedad y causalidad. En este sentido, el análisis de la estacionariedad es fundamental para la estimación de los pa-
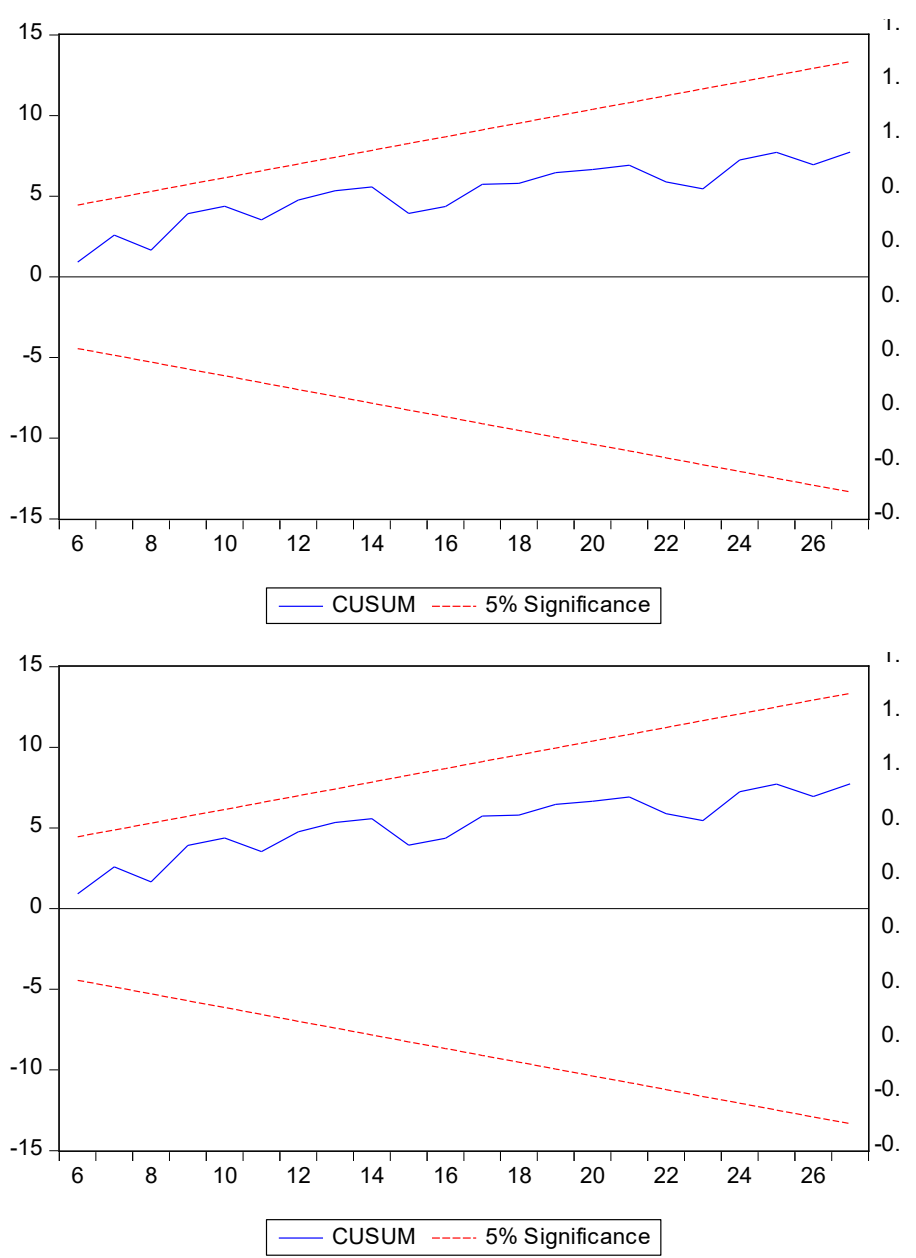

Figura 3. Pruebas CUSUM y CUSUMQ.

Fuente: elaboración propia a partir de E-views ${ }^{\circledR 9}$. 
rámetros cuando se aplica la regresión de mínimos cuadrados, una vez que se puede resultar en estimaciones de parámetros falsos (Presno y López, 2001). Además, es un factor el cual permite realizar predicciones precisas y su análisis debe ser efectuado antes de determinar la relación de causalidad entre variables (Diebold y Kilian, 2000).

El contraste de estacionariedad conocido como KPSS, el cual responde a los nombres de sus autores (Kwiatkowski, Phillips, Schmidt y Shin, 1992), es, sin duda, la referencia básica en este contexto. La prueba utiliza una hipótesis nula de estacionariedad y para el presente estudio, se emplearon dos especificaciones; una con sólo la constante y otra con constante y tendencia. Es de mencionar que, la prueba KPSS se puede considerar más adecuada debido a que ésta presenta mayor potencia frente a otras pruebas tradicionales (Melo y Misas, 1998). De acuerdo a los resultados presentados en la Tabla 6 el valor estadístico de las pruebas KPSS en todos los casos es menor al valor crítico para el nivel de significancia del 1\%, 5\% y 10\% (con excepción del CP y REND para el 10\%), por lo cual aceptamos la hipótesis nula de estacionariedad. Es de señalar, que la comprobación individual de las series se convierte en una condición suficiente de estacionariedad del modelo (Melo y Misas, 1998).
En el mismo orden de ideas, la noción de causalidad de Granger (1969) es importante en la econometría aplicada. La existencia de correlación entre dos variables no implica causalidad, esto es, que una variable se correlacione con otra no implica que una de ellas sea la causa de la alteración en los valores de la otra (Gujarati y Porter, 2010). La esencia de esta definición es sencillamente que, existe una relación entre dos variables, sin embargo, no explica el sentido de dicha relación. Es posible que X cause Y; también es posible que $\mathrm{Y}$ cause $\mathrm{X}$, y también lo es que tanto $\mathrm{X}$ como $\mathrm{Y}$ sean independientes.

En referencia a lo anterior la prueba de causalidad de Granger considera las siguientes hipótesis:

$$
\begin{aligned}
& \text { Ho: }=a_{1}=a_{2}=\cdots \cdots=a_{n}=0 \\
& \text { Ho: }=\theta_{1}=\theta_{2}=\cdots \cdots=\theta_{n}=0
\end{aligned}
$$

- Si al usar el estadístico F de mínimos cuadrados se rechaza la primera hipótesis nula $(p<0,05)$, pero no se rechaza la segunda hipótesis nula $(\mathrm{p}>0,05)$, se concluye que $\mathrm{X}$ causa a $\mathrm{Y}$ (causalidad unidireccional).

- Si al usar el estadístico F de mínimos cuadrados no se rechaza la primera hipótesis nula $(\mathrm{p}>0,05)$, pero se rechaza la segunda hipótesis nula $(p<0,05)$, se concluye que Y causa a X (causalidad unidireccional).

TABLA 6

Valores estadísticos y críticos para las pruebas KPSS

\begin{tabular}{llcccc}
\hline Variable & & Valor Estadístico & \multicolumn{3}{c}{ Valores Críticos } \\
\hline \multirow{2}{*}{ CP } & Solo constante & & $1 \%$ & $5 \%$ & $10 \%$ \\
& Con constante y con tendencia & 0,151 & 0,739 & 0,463 & 0,347 \\
\multirow{2}{*}{ REND } & Solo constante & 0,133 & 0,216 & 0,146 & 0,119 \\
& Con constante y con tendencia & 0,135 & 0,739 & 0,463 & 0,347 \\
\multirow{2}{*}{ I } & Solo constante & 0,231 & 0,216 & 0,146 & 0,119 \\
& Con constante y con tendencia & 0,066 & 0,739 & 0,463 & 0,347 \\
\multirow{2}{*}{ EDAD } & Solo constante & 0,055 & 0,216 & 0,146 & 0,119 \\
& Con constante y con tendencia & 0,058 & 0,739 & 0,463 & 0,347 \\
\hline \hline
\end{tabular}

Fuente: elaboración propia a partir de E-views ${ }^{\circledR} 9$ (2016). 
- Si al usar el estadístico F de mínimos cuadrados se rechazan las dos hipótesis nulas $(p<0,05)$, se concluye que las variables se causan mutuamente (causalidad bilateral).

- Si al usar el estadístico F de mínimos cuadrados no se rechazan las dos hipótesis nulas $(p>0,05)$, se concluye que la $\mathrm{X}$ y Y son independientes.

Como se observa en la Tabla 7 al aplicar la prueba de Granger entre todas las variables del estudio se obtuvo los valores $\mathrm{F}$ y sus respectivas probabilidades. Con relación a las variables EDAD y REND, así como EDAD e I, los valores estimados fueron significativos en el nivel de $5 \%$ donde la dirección de causalidad va de la EDAD al REND y de la EDAD al I, respectivamente. Esto indica una causalidad unidireccional, en otras palabras, se concluye que la edad del cultivo influye en el rendimiento y los ingresos de los productores, el cual puede ser explicado una vez que los árboles de durazno de las fincas estudiadas poseen una edad promedio de 4 años, es decir, se encuentran en una etapa ascendente de producción.

Igualmente se destaca que los demás resultados obtenidos del $\mathrm{F}$ estadístico son mayores que un alfa del $5 \%$, el cual indica que los conjuntos de coeficientes no son estadísticamente significativos en ambas direcciones, lo que implica que las variables son consideradas independientes.

En este sentido, podemos concluir que los resultados obtenidos para dichas pruebas validan las hipótesis del modelo quedando, así, especificado:

$$
\begin{gathered}
\mathrm{RENT}=74,18-4,84 \mathrm{E}-05 \mathrm{CP}+0,008916 \mathrm{REND}+ \\
6,68 \mathrm{E}-06 \mathrm{I}+71,59720 \mathrm{EDAD}
\end{gathered}
$$

Respecto a la especificación del modelo se obtiene que, en relación al costo de producción, tal como se esperaba, a medida que se incrementan la rentabilidad disminuye.

TABLA 7

Prueba de causalidad de Granger entre las variables

Pairwise Granger Causality Tests

Sample: 27

Lags: 2

\begin{tabular}{llll}
\hline Null Hypothesis & Obs & F-estadístico & Probabilidad \\
\hline I does not Granger Cause REND & 25 & 1,81483 & 0,1887 \\
REND does not Granger Cause I & & 1,21810 & 0,3168 \\
EDAD does not Granger Cause REND & 25 & 4,00619 & 0,0344 \\
REND does not Granger Cause EDAD & & 0,42940 & 0,6568 \\
CP does not Granger Cause REND & 25 & 0,50358 & 0,6118 \\
REND does not Granger Cause CP & & 0,68963 & 0,5133 \\
EDAD does not Granger Cause I & 25 & 4,09398 & 0,0323 \\
I does not Granger Cause EDAD & & 1,02810 & 0,3758 \\
CP does not Granger Cause I & 25 & 0,18882 & 0,8294 \\
I does not Granger Cause CP & & 1,16075 & 0,3335 \\
CP does not Granger Cause EDAD & 25 & 2,64351 & 0,0958 \\
EDAD does not Granger Cause CP & & 1,19589 & 0,3232 \\
\hline
\end{tabular}

Fuente: elaboración propia a partir de E-views ${ }^{\circledR 9}$ (2016). 


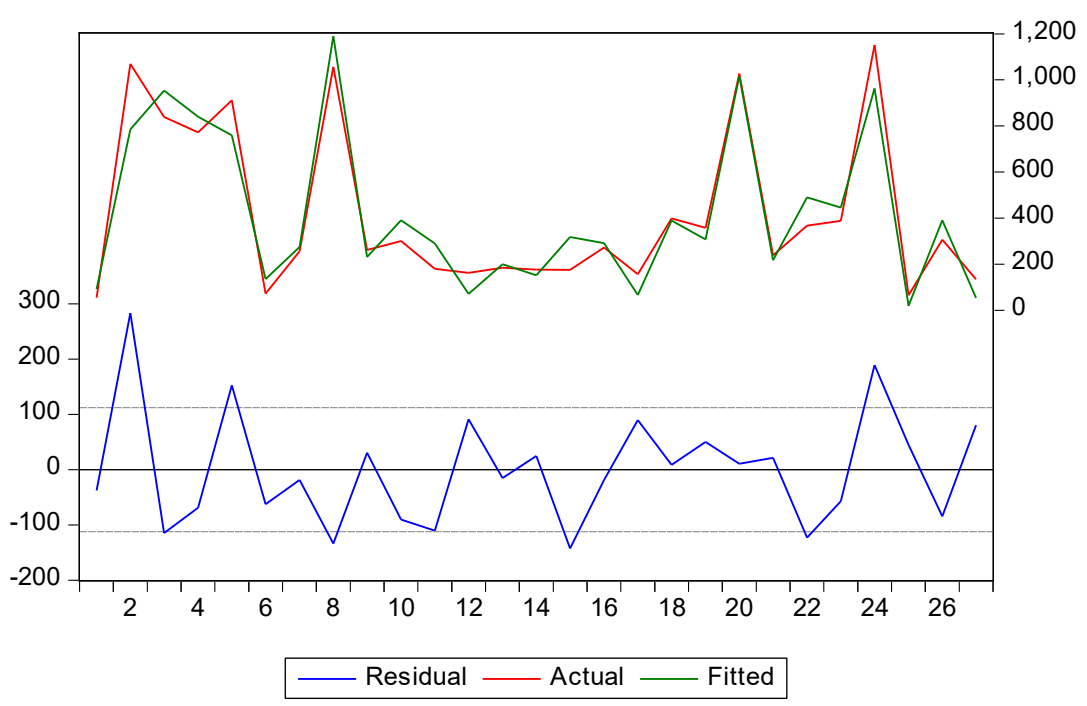

Figura 4. Datos del modelo de regresión lineal por MCO.

Fuente: elaboración propia a partir de E-views®9 (2016).

Cuanto a las demás variables (REND, I y EDAD) estas presentaron relaciones directas con la variable dependiente la cual valida el modelo planteado.

Por consiguiente, cuando el costo de producción aumenta en una unidad, la rentabilidad económica del cultivo de durazno disminuye en 0,000048 por ciento, mientras que, al aumentar el rendimiento del cultivo en una unidad la rentabilidad económica aumenta en 0,00891, ahora, cuando el ingreso del productor aumenta en una unidad monetaria la rentabilidad del cultivo aumenta en 0,00000668 por ciento. A medida que la edad del árbol de durazno se incrementa en un año la rentabilidad aumenta en $71 \%, c e$ teris paribus para los demás factores de producción. Es de resaltar que el intercepto, es decir la constante 74,18, no posee relevancia económica (Gujarati y Porter, 2010). Finalmente, la Figura 4 permite concluir que el modelo proyectado se ajusta bien a los datos.

\section{Conclusiones}

El cultivo de durazno en la provincia de Pamplona es una importante fuente económica para los agricultores, por ser una activad intensiva en mano de obra, que genera empleo, así como, oportunidades de negocio (Puentes et al., 2015). La mayor parte de la producción proviene de pequeños productores heterogéneos $(76,3 \%)$, en todo sentido, incluyendo lo económico, el grado de desarrollo tecnológico y comercial (Villamizar, 2008).

Por lo consiguiente, se propuso desarrollar un modelo explicativo sobre la rentabilidad económica del cultivo de durazno de veintisiete (27) productores en la provincia de Pamplona, una vez que, desde una perspectiva económica, permite a los productores planear y gestionar de manera eficiente las unidades productivas.

En vista de lo anterior, se estima la rentabilidad económica, tomando como referencia los costos de producción, rendimiento, ingresos y edad. Los resultados comprobaron que los coeficientes de regresión del modelo son significativamente diferentes de cero, a un nivel de significancia del $5 \%$, por lo tanto, las variables explicativas (CP, REND, I y EDAD) contribuyeron a explicar individualmente la variable RENT. Igualmente, se efectuaron pruebas con la finalidad de verificar la validez del modelo propuesto, del cual se acepta el supuesto de normalidad, además, no se evidencian problemas de colinealidad, heteroscedasticidad y autocorrelación 
Por otra parte, los modelos econométricos llevan implícito el supuesto de constancia de los parámetros para todos los individuos. Para tal fin, se examina la existencia de la estabilidad de los estimadores a través de las pruebas CUSUM y CUSUMQ y se corrobora la estabilidad de los mismos. De igual modo las pruebas de KPSS y de Granger indicaron la presencia de estacionariedad y una causalidad unidireccional desde la variable EDAD hacia el REND y de EDAD hacia el I, lo cual indica que la edad del cultivo de durazno influye en el rendimiento y los ingresos del cultivo de durazno.

Finalmente, se concluye, según los datos obtenidos, que el modelo se ajusta a lo propuesto inicialmente, es decir, las variables CP, REND, I y EDAD explican en gran parte la RENT, siendo así, una herramienta útil para los productores de durazno de la región al identificar de manera confiable las variables que afectan la rentabilidad económica, y de esta forma, optimizar los recursos de forma eficiente.

Los autores expresan sus agradecimientos a la Universidad de Pamplona por el financiamiento de esta investigación proyecto interno titulado: Aporte al estudio de la huella genómica mediante marcadores moleculares SSR de Prunus persica (Var. amarillo Jarillo y gran jarillo), y el patógeno Monilinia fruticula en cultivos comerciales de durazno en la provincia de Pamplona.

\section{Referencias}

Africano, K., Almanza-Merchón, P., Criollo, H., Herrera, A. y Balaguera-López, H. (2016). Caracterización poscosecha del fruto de durazno $P$. pérsica (L.) Batsch cv. Dorado producido bajo condiciones de trópico alto. Revista Colombiana de Ciencias Hortícolas, 10(2), 232-240.

Aguirre, J., Prieto, M. y Escamilla, J. (1997). Contabilidad de costos, gestión y control presupuestario, control de gestión, la función del controller. Madrid: Cultural de Ediciones.
Cárdenas, J. y Fischer, G. (2013). Clasificación botánica y morfología de manzano, peral, duraznero y ciruelo. En: Fischer y Carranza (Eds), Los frutales caducifolios en Colombia: Situación actual, sistemas de cultivo y plan de desarrollo (24-25). Bogotá, D.C.: Sociedad Colombiana de Ciencias HortícolasSCCH.

Campos, T. (2013). Especies y variedades de hoja caduca en Colombia. En: Fischer y Carranza (Eds), Los frutales caducifolios en Colombia: Situación actual, sistemas de cultivo y plan de desarrollo (47-65). Bogotá, D.C.: SCCH.

Diebold, F. y Kilian, L. (2000). Unit-Root tests are useful for selecting forecasting models. Journal of Business and Economic Statistics, 18(3), 265-273.

E-views (Version 9.0) [Software]. (2016). Irvine: IHS Global Incorporation. Recuperado de www.eviews.com

Faga, H. y Ramos, M. (2006). Como profundizar en el análisis de sus costos para tomar mejores decisiones empresariales. Buenos Aires: Granica.

Fischer, G. (2013). Comportamiento de los frutales caducifolios en el trópico En: Fischer y Carranza (Eds), Los frutales caducifolios en Colombia: Situación actual, sistemas de cultivo y plan de desarrollo (31-46). Bogotá, D.C.: Sociedad Colombiana de Ciencias Hortícolas.

Flórez, J. y Miranda, E. (2017). Factores que influyen en la rentabilidad económica de la producción del cultivo de camu camu en la selva peruana. Revista Tzhoecoen, 9(1), 94-106.

Gómez, M. y Rodríguez, J. (2007). Análisis de estabilidad en variables económicas de México. Revista Nicolaita de Estudios Económicos, 2(2), 9 - 30

Granger, C. (1969). Investigating Causal Relations by Econometric Models and Cross-spectral Methods. Econometrica, 37(3), 424-438. 
Gujarati, D.y Porter, D. (2010). Econometría. México, D.F.: McGraw Hill.

Jarque, C. y Bera, A. (1987). A test for normality of observations and regression residuals. International Statistical Review, 55,163-172.

Kleinbaum, D., Kupper, L. y Muller, K. (2014). Applied Regression analysis and other multivariables methods (5th ed.). Boston: Cengage Learning.

Kwiatkowski, D., Phillips, P., Schmidt, P. y Shin, Y. (1992). Testing the null hypothesis of stationarity against the alternative of a unit Root: How sure are that economic time series have a unit root. Journal of Econometrics, 54(1-3), 159-178.

Melo, L. y Misas, M. (1998). Análisis del comportamiento de la inflación trimestral en Colombia bajo cambios de régimen: Una evidencia a través del modelo "Switching" de Hamilton. Revista de Economía del Rosario, 1(2), 91-112.

Patiño, L. y Miranda, D. (2013). Situación actual de los frutales caducifolios en el mundo y en Colombia. En: Fischer y Carranza (Eds), Los frutales caducifolios en Colombia: Situación actual, sistemas de cultivo y plan de desarrollo (9-20).

Pinzón, E., Cruz, M. y Fischer, G. (2014). Aspectos fisiológicos del duraznero $(P$. persica (L.) Batsch) en el trópico alto. Revista UDCA Actualidad \& Divulgación Científica, 17(2), 401- 411.

Peñaranda, G. (2012). Análisis de los costos de la producción de durazno P. pérsica en la Provincia de Pamplona, Norte de Santander. Revista Face, 1, 145-162.

Presno, J. y López, A. (2001). Estacionariedad en torno a un nivel con ruptura. Un estudio de simulación. Estudios de Economía Aplicada, 18, 189-208.

Puentes, G., Bastidas, L. y Lemus, Á. (2015). Estimación de pérdidas técnicas y financieras en cosecha de durazno, en la granja Tunguavita de la U.P.T.C. Boyacá. Revista Agronomía, 23(1), 93-105.
Quevedo, E., Darghan, A. y Fischer, G. (2017). Clasificación de variables morfológicas del duraznero (Prunus persica L. Batsch) 'Jarillo' en la montaña santandereana colombiana mediante análisis discriminante lineal. Revista Colombiana de Ciencias Hortícolas, 11(1), 39-47.

Ramírez, C. y Ávila, L. (2013). Modelo para medir la rentabilidad de los cereales alto andino en el departamento de Puno caso quinua. Revista de Investigación Altoandina, 15(1), 187-199.

Ramírez, H. y Vanegas, B. (2008). Gestión de costos en las PYMES: problemática y alternativas de solución. Bogotá, D.C.: Universidad Libre.

República de Colombia. Ministerio de Agricultura de Colombia. (2014). Banco de datos Agronet. Recuperado de http:// www.agronet.gov.co/estadistica/Paginas/ default.aspx.

Rivero, P. y Cuervo, J. (1986). El análisis económico-financiero de la empresa. Revista Española de Financiación y Contabilidad, 49,15-33.

Thadewald, T. y Büning, H. (2007). JarqueBera Test and its competitors for testing normality - A power comparison. Journal of Applied Statistics, 34, 87-105.

Varían, H. (2016). Microeconomía Intermedia: Barcelona: Antoni Bosch.

Villamizar, E. (2008). El durazno: un proceso de cambio en la agricultura de la Provincia de Pamplona. Revista Face, 11(1), 189-203.

Enrique Quevedo García es Agrónomo, MSc. Fisiología de Cultivos, Docente Titular, Facultad Ciencias Agrarias,

Giovanni Cancino Escalante es Biólogo, PhD Biotecnología, Docente Titular, Facultad Ciencias Básicas,

Susan Cancino es Economista, MBA, Docente, Facultad Ciencias Económicas y Empresariales, 